Tomasz Kaczmarek

Université de Łódź

http://dx.doi.org/10.18778/8088-896-8.20

\title{
LA VOIX DES FEMMES DANS LE THÉÂTRE FRANÇAIS DE CONTESTATION SOCIALE AU TOURNANT DU XX ${ }^{\mathrm{E}}$ SIÈCLE
}

L'anarchie symbolise la libération de l'esprit humain de l'aliénation de la religion, la libération $\mathrm{du}$ corps humain de la domination de la propriété, la libération des chaînes qui nous lient à l'oppression des gouvernements. Elle défend un ordre social fondé sur la libre association entre individus.

Emma Goldman

Voici la lutte universelle :

Dans l'air plane la Liberté !

A la bataille nous appelle

La clameur du déshérité !...

... L'aurore a chassé l'ombre épaisse,

Et le Monde nouveau se dresse

Al'horizon ensanglanté !

Louise Michel

\section{En guise d'introduction}

Pendant plus d'une centaine d'années peu de gens sont au courant de l'existence du théâtre féministe en France vers la fin du XIX ${ }^{\mathrm{e}}$ siècle, la majorité écrasante étant convaincue qu'il est né suite aux événements perturbateurs de $1968^{1}$. Ce théâtre a été condamné à l'oubli pour des raisons politiques évidentes. On l'accusait, il est vrai, d'un dilettantisme n'ayant rien à voir avec un « vrai art », ce qui est

1 M.-C. Pasquier, M.-C. Rouyer, M. Surel-Tupin, « Théâtre féministe », [in] Dictionnaire encyclopédique du théâtre (dir. M. Corvin), Larousse, 2001. 
partiellement juste, mais la production dramatique qui raillait sans pitié les dominants de ce monde, devait déranger profondément le pouvoir, surtout quand les femmes écrivains se sont fait entendre au sein d'une mouvance ouvertement anarchiste. Snobé par les critiques et chercheurs, ce théâtre semblait enseveli à jamais. La situation change en 1991 quand on publie la première anthologie du théâtre de contestation sociale ${ }^{2}$, dans laquelle à côté de dramaturges tels que Georges Darien, Lucien Descaves, Octave Mirbeau ou Jean Grave, apparaît le nom de Vera Starkoff, militant farouchement pour l'égalité des sexes. Une seconde anthologie, en trois volumes, remporte un succès retentissant, dans laquelle, mise à part le dramaturge d'origine russe évoquée ci-dessus, on peut découvrir d'autres femmes engagées qui critiquent avec une verve révolutionnaire les absurdités de l'ordre social tout en attirant l'attention sur la condition du beau sexe dominé par un patriarcat égoïste et pervers ${ }^{3}$. Il s'agit de Louise Michel et de Nelly Roussel dont on chercherait en vain les noms dans les manuels dédiés à la littérature et au théâtre. Grâce à ces deux publications, le public a eu enfin la chance non seulement de prendre connaissance du théâtre de propagande au tournant du $\mathrm{XX}^{\mathrm{e}}$ siècle, mais aussi d'apprécier les ouvrages de femmes qui, tout en restant minoritaires parmi les « parias » de la société, s'érigeaient en amazones combatives. Dès lors, l'intérêt que les chercheurs portent pour ce type de théâtre s'accroît toujours. Cecilia Beach écrit un livre bien documenté sur les œuvres des femmes qui écrivent en France à une époque de grands bouleversements sociaux, son étude étant focalisée sur la perspective des théories du genre ${ }^{4}$. Séverine Auffret a donné au Théâtre du Rond-Point une conférence fort intéressante le 19 novembre 2009 sur le théâtre de combat de Vera Starkoff, l'une des premières femmes qui ait écrit pour la scène et fondatrice hors pair des universités populaires. Philippe Ivernel, pour sa part écrit un article consacré à Louise Michel dans les colonnes du périodique Romantisme ${ }^{5}$. Nous consacrons ces quelques lignes aux deux dramaturges : Starkoff et Roussel, qui ont explicitement abordé la problématique du rôle de la femme dans la société bourgeoise. Quant à Louise Michel, elle se fait le porte-drapeau des anarchistes et son attention se concentre sur les luttes sociales au sens large du mot, c'est pourquoi elle mérite une étude à part.

${ }^{2}$ Le théâtre de contestation sociale autour de 1900, textes réunis et présentés par J. Ebstein, J. Hughes, Ph. Ivernel. M. Surel-Tupin, PUBLISUD, 1991.

${ }^{3}$ Au temps de l'anarchie, un théâtre de combat : 1880-1914, choix et éditions des textes par J. Ebstein, Ph. Ivernel, M. Surel-Tupin et S. Thomas, Séguier/Archimbaud, 2001, 3 volumes.

${ }^{4}$ C. Beach, Staging Politics and Gender, French Women's Drama, 1880-1923, New York, Palgrave Macmillan, 2005.

${ }^{5} \mathrm{Ph}$. Ivernel, « Romantisme révolutionnaire et Réalisme paroxystique. Théâtre de Louise Michel », « Rejet et renaissance du romantisme à la fin du XIX ${ }^{\mathrm{e}}$ siècle », Romantisme, $\mathrm{n}^{\circ} 132$. 


\section{Les luttes de Vera Starkoff}

Vera Starkoff est une femme énigmatique dont on sait peu de choses. Néanmoins, grâce à l'étude approfondie de Cecilia Beach nous apprenons qu'elle est née le $1^{\text {er }}$ avril 1867 à Vilnius en tant que Tauba Efron. Comme la majorité des jeunes femmes russes provenant des couches aisées, la future anarchiste fait ses études en Suisse puis, vers la fin du XIX ${ }^{\mathrm{e}}$ siècle, se rend à Paris, terre promise des réfugiés russes, des révolutionnaires polonais et des juifs fuyant les pogromes. Elle y résidera jusqu'à sa mort en 1923. Harcelée par la police tsariste, Tauba se cache sous le pseudonyme de Starkoff et se lance dans les luttes sociales. Admiratrice de Tolstoï, elle promeut son œuvre libertaire à l'étranger et bientôt écrit ellemême des textes dans lesquels elle arrache les masques d'un système capitaliste inhumain. Elle rejette toutes les violences et les dogmes religieux qui garrottent la liberté des individus. En propageant les universités populaires où les pauvres, souvent illettrés, peuvent participer aux conférences consacrées aux inégalités de la société bourgeoise, suivies habituellement de spectacles, elle pense aussitôt à une nouvelle expression théâtrale qui puisse illustrer ses idées progressistes. L'une d'elles concerne la condition instable de la femme dans le régime patriarcal. De fait, elle lutte pour le théâtre ouvert à tout le monde, le théâtre populaire qui aborde les vrais problèmes du peuple :

en affirmant le droit de tous au théâtre, il [le théâtre populaire] élargira forcément l'horizon scénique, et nous verrons d'autres sujets de pièces que les histoires amoureuses de marionnettes mondaines, d'autres personnages que des mannequins de salons destinés à amuser les fêtards oisifs, incapables de supporter un spectacle qui fasse penser. Le Théâtre Populaire nous ramènera aux grands sujets qui intéressent la masse tout entière : la joie et la souffrance, la vie et la mort, les conditions et les rapports des hommes, le besoin d'idéal qui est la source principale du progrès ${ }^{6}$.

L'un des problèmes qui préoccupe Starkoff, c'est la vocation de la femme dans la société moderne et surtout sa place dans le couple. Au début du XX siècle la question de l'amour libre est à l'ordre du jour et fait couler beaucoup d'encre (pas toujours le meilleur) dans les journaux de l'époque. Plusieurs auteurs anarchistes se sont exprimés sur cette problématique, mais malgré des voix parfois divergentes, tous semblent insister sur l'iniquité d'un système bourgeois périmé qui condamne avant tout la femme au joug du mariage ( $\ll$ Le mariage, c'est la chaîne qui retient l'homme et la femme prisonniers l'un de l'autre $\rangle^{7}$ ). La voix de Madeleine Vernet est à ce propos exemplaire. Il est intéressant d'évoquer ici p. 12.

${ }^{6}$ V. Starkoff, « Lettre », Revue d'art dramatique, janvier-décembre 1906, tome XVI, Madeleine Vernet, « Libre amour », Éditions de l’Anarchie, 1907. 
les grandes lignes de ses réflexions sans aucun doute partagées par Starkoff en la matière. La militante pacifiste attire notre attention sur le fait très important que l'amour est fugace tel un «enfant de bohème » et personne n'est à même de garantir la stabilité de ce sentiment. L'institution du mariage ne répond pas aux besoins de l'amour, mais le pervertit. Or, « qu'on ne confonde pas l'amour avec le mariage. Le mariage, c'est une convention sociale ; l'amour, c'est une loi naturelle. Le mariage, c'est un contrat ; l'amour, c'est un baiser. Le mariage, c'est une prison ; l'amour, c'est un épanouissement. Le mariage, c'est la prostitution de l'amour $\gg^{8}$. Vernet est contre la monogamie qui lie l'homme à la femme d'une manière artificielle, dès lors il faut émanciper les sentiments interhumains de toutes les contraintes aussi conventionnelles qu'aberrantes :

Pour qu'il conserve sa beauté et sa dignité, l'amour doit être libre ; et il ne peut être libre que s'il est régi par une seule loi. Il ne peut y avoir sur ce chapitre de considérations d'ordre matériel ou moral : deux êtres s'aiment, se désirent, se le disent ; ils doivent avoir le droit de se donner l'un à l'autre sans que nulle raison étrangère à leur désir n'intervienne entre eux, comme ils doivent avoir le droit le plus absolu de se quitter le jour où ils ne se désirent plus?.

L'immoralité, dont Vernet pourrait être facilement accusée suite à ses propos, n'est, à ses yeux, qu'une obligation perverse qui force l'homme à observer des règles conventionnelles; ces règles étant un obstacle cruel et pathologique pour l'efflorescence libre de l'être humain. De fait, l'immoralité est contre nature comme les épousailles mercantiles : « Mais l'amour libre ne peut être de l'immoralité puisqu'il est une loi naturelle ; le désir sexuel ne peut être une immoralité puisqu'il est un besoin naturel de notre vie physique $\gg$ comme la faim ou

8 Vernet dans son texte compare à plusieurs reprises le mariage à la prostitution : « J'ai dit que l'amour doit être absolument libre, aussi bien pour la femme que pour l'homme. Et j'ajoute encore : l'amour ne peut véritablement exister qu'à la condition d'être libre. Sans la liberté absolue, l'amour devient de la prostitution, de quelque nom qu'on le revêt. Le fait de vendre son corps à un prix plus ou moins élevé, à une nombreuse clientèle, ne constitue pas seulement la prostitution. La prostitution n'est pas seulement l'apanage de la femme, l'homme aussi se prostitue. Il se prostitue quand, dans le but d'un intérêt quelconque, il donne des caresses sans en éprouver le désir. Non seulement le mariage légal est une prostitution lorsqu'il est une spéculation de l'un des époux sur l'autre, mais il est toujours une prostitution puisque la vierge ignore ce qu'elle fait en se mariant. Quant au devoir conjugal, ce n'est ni plus ni moins encore que de la prostitution ; prostitution, la soumission au mari ; prostitution, la résignation et la passivité. Prostitution encore que l'union libre, quand elle passe de l'amour à l'habitude. Prostitution enfin, tout ce qui rapproche les sexes en dehors du désir et de l'amour » (Ibidem).

\section{Ibidem.}


le sommeil. Ces idées novatrices, qui pourraient même aujourd'hui en ébranler quelques-uns, ont envenimé les esprits à tel point qu'elles demandèrent à être immortalisées par le théâtre.

En s'inspirant de ce débat animé, Starkoff compose une pièce vivante dans laquelle elle expose ses idées libertaires. Elle retrace l'une des soirées ouvrières auxquelles son œuvre est au demeurant dédicacée. De fait, la scène s'ouvre sur la bibliothèque derrière laquelle se trouve la salle de conférences de l'université populaire. C'est là où s'entretient Blanche, une jeune secrétaire, avec Ruinet, un conférencier qui doit se prononcer sur le problème de l'amour libre. C'est dans cet endroit que nous voyons défiler plusieurs personnages qui désirent participer à la conférence annoncée. Parmi eux on retrouve, entre autres, le couple ouvrier aux idées sociales, le travailleur consciencieux et l'ouvrier misogyne. En peuplant sa pièce de ces comparses, Starkoff veut tout simplement décrire fidèlement les rencontres entre les gens de diverses opinions que regroupe l'UP, mais l'intrigue principale se concentre sur la question du rôle de la femme dans le couple. C'est autour de ce sujet (le pivot essentiel du drame) que tourne la conversation entre Blanche et Ruinet. Ce dernier, malgré une incontestable ouverture d'esprit, constate inopinément à un moment donné que « dans l'union libre l'homme est plutôt victime que bourreau; la femme est plus infidèle de nature $\gg^{10}$. Pour contrecarrer cette conviction, l'humble brodeuse raconte l'histoire de sa vie qui illustre bien les injustices imposées au beau sexe par la société phallocentrique. Son père, un banquier riche, abandonne sa famille pour une jeune actrice : de cet épisode découlent tous les malheurs de sa vie future. Sa mère connait des revers de fortune, rejette l'hypocrisie de la classe bourgeoise de laquelle elle provient et se consacre corps et âme à l'éducation de sa petite fille. Au moment de mourir prématurément, la mère prévient sa fille de se méfier des hommes. Nonobstant ces précautions, Blanche se fait séduire par un jeune homme, fraîchement licencié en droit, avec lequel elle aura un enfant. Les promesses du bourgeois d'aimer sincèrement la femme et de vouloir l'épouser s'avèrent aussitôt mensongères, car il est fiancé avec une autre depuis deux ans. Déçue et complètement abattue, Blanche désire se venger et révéler toute la vérité à l'épouse « légalement » promise. Cependant, dès qu'elle la rencontre, elle oublie sa détresse et revient sur sa décision. Elle ne veut pas briser la vie de la jeune fille que l'homme va prendre pour femme et voue tout son amour à l'enfant dont elle va accoucher. Le conférencier est indigné par ses propos et traite l'homme de lâche impardonnable, sa rage gronde encore plus quand il apprend que le séducteur se présente à l'UP étant donné qu'il prépare les élections. Sur ce point, Ruinet se dit prêt à punir l'homme volage au cours de son intervention, mais Blanche, émue, supplie son camarade de ne rien faire, car elle pourrait perdre l'être le plus cher pour elle qu'est son bébé.

${ }^{10}$ V. Starkoff, L'Amour libre, [in] Au temps de l'anarchie..., p. 307. 
La femme lui explique que le traitre se dévoilera tout seul puisque les ouvriers de l'UP ne se laissent pas leurrer par de belles phrases, la conscience publique étant, selon elle, un tribunal pour ce politicien carriériste. Et de fait, une fois à la tribune, Louine (le séducteur) est dénigré par les rassemblés qui ne veulent pas entendre les balivernes du bourgeois. C'est ainsi que Blanche a été vengée. Bouleversé par la sagesse de la brodeuse, Ruinet semble changer d'opinion sur l'amour libre. Quoique nous n'allions pas entendre ses propos, car la pièce finit juste avant sa prise de parole, Starkoff a suffisamment exprimé ses idées sur la question à travers Blanche. C'est elle qui exhorte à la résiliation du joug législatif injurieux : «abolissez donc la loi qui proclame la bâtardise, donnez à tous les enfants le même droit au bonheur, et vous détruirez la misère! Et la maternité ne sera plus une chaîne, mais un titre de gloire, l'amour - un épanouissement de vie ! $\gg^{11}$ Sans aucun doute cette pièce aurait pu jouir d'un certaine renommée à une époque de grands bouleversements sociaux, mais de nos jours, son aspect profondément didactique aurait du mal à rencontrer l'auditoire qu'elle mérite.

Dans la deuxième pièce, L'Issue (1904), qui a connu un succès indéniable auprès du public fréquentant les universités populaires, on découvre facilement la verve de la plume d'Ibsen. De fait, Starkoff semble retranscrire à sa manière l'histoire de Nora, hérö̈ne du fameux drame du Norvégien en l'agrémentant tout de même d'accents plus révolutionnaires. En peu de mots, la Russe présente l'histoire d'une jeune fille provenant d'une famille aisée, Lucie, qui sous l'emprise de Roche, instituteur et membre de l'UP, subit une métamorphose et qui, d'une demoiselle bien polie connaissant et respectant les mœurs de la classe prépotente, devient une rebelle contre la moralité des bourgeois, symbolisés dans la pièce par ses parents. En écrivant son œuvre, Starkoff pense bien évidemment aux spectateurs qu'elle désire éclairer sur le statut présumé inférieur de la femme dans une société dominée par les hommes. C'est pour cette raison que dans les deux actes elle veut opposer les deux mondes : bourgeois et ouvrier. Ce premier (acte I) est reflété par un intérieur prétentieux de mauvais goût, dans lequel on remarque le manque de livres tandis que le deuxième (acte II), malgré son aspect modeste, est propre avec un peu partout des livres. Ce manichéisme simpliste est un procédé efficace qui, dès le début de la pièce, annonce qui est l'ennemi du progrès social.

L'action du premier acte se déroule ainsi dans la maison bourgeoise où règne sans partage le pater familias désireux que tous sauf exception obtempèrent aveuglément à sa volonté que l'on pourrait aujourd'hui qualifier de « machiste ». Il impose surtout son pouvoir à ses deux enfants qu'il traite comme de vrais esclaves, sa femme étant depuis longtemps « apprivoisée » par sa volonté autocratique. Cependant, les premières failles de la sérénité autoritaire se manifestent en fonction de la prise de conscience de la progéniture. La fille se montre plus par-

${ }^{11}$ Ibidem, p. 309. 
ticulièrement récalcitrante à l'oppression paternelle. Or, passée l'âge de 22 ans, Lucie est promise à un « homme d'affaires », mais la jeune fille n'aime pas monsieur Toudoux malgré sa docilité incontestable et les sentiments sincères qu'il exprime envers sa promise. Conformément à la vulgate grossièrement conformiste, ceci ne pose aucun problème du point de vue sentimental, car le mariage n'est, comme aurait dit Wedekind, qu' un simple contrat commercial - telles sont aussi les convictions du dramaturge en la matière. Ne pouvant plus supporter ses géniteurs oppressifs, la jeune femme pense à échapper à la cage dorée dans laquelle elle étouffe. Même si elle envisage momentanément le fait que le mariage pourrait l'aider à réaliser ses plans, elle se rend compte aussitôt qu'en épousant un homme choisi par ses parents, elle n'atteindrait pas la liberté convoitée, mais qu'elle changerait uniquement de propriétaire. Sa vraie issue serait de tourner définitivement le dos à ce monde bigot et mesquin dans lequel elle se sent comme une prisonnière. Elle ne peut même pas compter sur sa mère qui admet la supériorité de son mari, car « dans nos églises - comme elle s'explique devant l'instituteur - on nous enseigne que le Seigneur a commandé l'humilité et la résignation $\gg^{12}$. Le jeune homme aux idées libertaires présente toute une autre exégèse de la Bible et souligne le caractère parfaitement subversif de l'activité de Jésus. Les propos de l'enseignant sapent, selon les parents, les fondements mêmes de la société bourgeoise, c'est pourquoi le père licencie rudement le libre-penseur, ce qui incite seulement la jeune femme à passer à l'acte. Lucie, instruite par Roche, décide de s'insurger contre le pouvoir paternel et quitte définitivement sa famille.

Nous la retrouvons dans le deuxième acte dans la demeure simple, mais accueillante du jeune éducateur. Celui-ci est surpris par la visite nocturne de la jeune fille. Il n'est pas mécontent, tant s'en faut, il en est amoureux depuis longtemps, mais, tout en restant profondément ému par cet acte, sans aucun doute courageux, de Lucie, il craint, du moins tout au début, que ce geste n'ait été tout simplement qu'un « coup de tête » et que le lendemain la demoiselle ne soit heureuse de retourner chez ses parents. Tout porterait donc à croire que Roche partage les convictions selon lesquelles les femmes agissent plutôt par émotion que par raison. De fait, on pourrait être déçu par sa réaction d'autant plus que l'homme demande, voire même exige de la femme, une épreuve, une sorte $\mathrm{d}^{\prime}$ « apprentissage de misère ». Roche n'est pas misogyne pour autant, il sait bien que la jeune femme n'a jamais travaillé et qu'il ne pourrait pas lui proposer la vie « bourgeoise » à laquelle elle était habituée. Et quand Starkoff étale devant nous le raisonnement du protagoniste, elle faillit par la tonalité aussi didactique qu'éloquente, qu'il est impossible de ne pas juger comme trop tarabiscotée. L'instituteur déclare comme un aumônier laïque : « je ne veux pas que l'amour vous

${ }_{12}$ V. Starkoff, L'Issue, [in] Au temps de l'anarchie..., p. 323. 
entraîne au travail, je veux que le travail vous élève jusqu'à l'amour $\gg^{13}$ et quelques instants plus tard quand il évoque la réalité austère de l'existence des pauvres, il continue en apôtre du marxisme :

c'est la tâche quotidienne et prosaïque de la vie matérielle et si secondaire qu'elle soit, on ne peut s'en décharger sur les autres sans retomber dans l'erreur séculaire, l'erreur qui divisait autrefois l'humanité en maîtres et en esclaves, qui les divise maintenant en patrons et domestiques. Les ouvriers actuels sont des esclaves déguisés ${ }^{14}$.

Si au début, la conversation semble s'inscrire dans l'esthétique réaliste, car elle prend en considération deux individus particuliers qui débattent sur les inégalités dans la société industrielle, vers la fin de cette-ci, nous avons l'impression que les personnages, comme c'était le cas dans le théâtre expressionniste, incarnent plutôt des idées et symbolisent respectivement le genre sexuel au détriment de leurs traits personnels. Ce n'est plus Roche qui s'entretient avec la belle Lucie, mais l'homme avec la femme. L'allégorisation des personnages permet à l'auteure de lancer un appel à la fraternité quelque peu utopique entre eux, mais son optimisme tourné vers l'avenir vise à donner de l'espoir, son but principal étant de favoriser la prise de conscience de tous les citoyens. Même si du point de vue formel, le drame fait penser à une œuvre naturaliste, il bascule sans aucun doute dans l'esthétique d'une pièce parfaitement militante, ce qui d'ailleurs sera confirmé dans le monologue final. De fait, sur un ton toujours pathétique, qui rappelle l'emphase typiquement expressionniste, Roche inculpe l'égoïsme masculin et glorifie la solidarité entre les sexes, sans laquelle la marche du monde n'a pas de chance d'avancer. Il ne le dit pas en son propre nom, mais en celui de tous les hommes, comme s'il était un représentant de ceux-ci :

L'homme, bêtement, s'est arrogé le monopole de la vérité. Il a enlevé à la femme ce qui compose l'essence même de la vie, la pensée et la liberté ! Il en a fait un cadavre, et sa cohabitation avec une morte les précipite tous deux dans la tombe! Il faut que l'homme relève sa compagne jetée à terre, pour qu'il reste debout ${ }^{15}$.

\section{La rage de Nelly Roussel}

À Roubaix un correspondant local relate : « les calotins ont tenté, dimanche dernier, de reprendre la rue, de faire revivre les mœurs du passé en processionnant. Mal leur en a pris, car nous lançâmes un manifeste invitant le peuple à ve-

\footnotetext{
13 Ibidem, p. 339.

14 Ibidem.

15 Ibidem, p. 340.
} 
nir protester contre ces menteurs, inquisiteurs et soutiens de la force $\gg^{16}$. Ces mots de Vera Starkoff caractérisent bien l'activité journalistique (elle aura à son actif plus de 200 articles) et dramatique de Nelly Roussel (1878-1922) qui, en tant que libre penseuse, se déclare de bonne heure partisane enfiévrée de l'anti-conception et de l'éducation sexuelle pour les jeunes filles, qui devrait être garantie par l'État. Elle se montre alors l'ennemie numéro un de la morale ecclésiastique. Elle partage avec Madeleine Pelletier la conviction que la femme doit décider pour elle-même surtout en ce qui concerne la question de la maternité, c'est pourquoi elle est en faveur d'une légalisation simple de l'avortement. Élevée dans une famille catholique, elle s'en éloigne très tôt, car elle voit dans les machinations de la religion une force millénaire qui réduit la femme à un simple objet sexuel. Suite à une logique aussi incompréhensible que libidineuse, la femme sera donc perçue comme la seule responsable du péché universel, elle en sera même son incarnation. Roussel est très éloquente et passe rapidement pour une oratrice incontournable, elle présente ses conférences en France et à travers toute l'Europe jouissant à chaque fois d'un accueil chaleureux par le public avide de ses interventions. Son mot d'ordre : « arrachez les femmes aux griefs de l'Église catholique $\gg^{17}$. Sa verve polémique trahit son faible pour l'expression dramatique. Au demeurant, elle adore le théâtre (ses auteurs favoris sont Jean Corneille, Victor Hugo) qui peut, selon elle, rallier plus efficacement l'audience à ses idées libérales. Ainsi que dans ses papiers elle se montre impitoyable, dans ses pièces sa plume fouette implacablement toute la tradition judéo-chrétienne.

Elle commence sa carrière de dramaturge par une très courte, mais fortement courageuse 《scène symbolique » Par la révolte qui a été représentée pour la première fois le $1^{\text {er }}$ mai 1903 et puis reprise au Théâtre Sarah-Bernhardt (1905). Ce dramaticule n'a rien de commun avec les idées ésotériques, chères aux écrivains symbolistes, tant s'en faut, car la pièce vise à dénoncer les piliers fondamentaux de la société qui oppriment depuis des siècles la femme. En modernisant l'ancien genre théâtral de la moralité, l'auteure désire convaincre d'autres femmes de se révolter contre les injustices millénaires qui leur sont infligées d'une manière absolument indécente. En d'autres mots, Roussel accuse les pouvoirs masculins représentés tant par les religieux que par les républicains n'épargnant personne faisant preuve d'idées rétrogrades. Au lever du rideau, nous voyons une pauvre Ève qui, une corde à la taille et des chaînes aux bras, est réduite à l'état d'esclave. Les cheveux épars, la femme reste au centre, agenouillée dans une attitude abattue, entourée des deux côtés de la scène par des personnages aussi fantasmagoriques

16 V. Starkoff, note dans Les Temps nouveaux, du 15 au 21 juin 1901, p. 2.

17 F. Rochefort, « Contrecarrer ou interroger les religions », [in] Le siècle des féminismes, E. Gubin, C. Jacques, F. Rochefort, B. Studer, F. Thébaud, M. Zancarini-Fournel (éds.), Paris, Les Éditions de l’Atelier, Éditions Ouvrières, 2004, p. 349. 
que monstrueux qui rappellent par leur solennité les personnages du théâtre de Jean Genet. La scène s'ouvre sur une espèce d'oratorio plaintif d'Ève au cours duquel elle se lamente sur sa condition d'asservissement cruel, pourtant considérée comme naturelle ${ }^{18}$. L’Église, enveloppée de longs voiles noirs, entourée de fleurs et de cierges, est sourde aux pleurs de la femme. Au contraire, elle s'avère impitoyable et sévère envers la première pécheresse : « Femme, créature impure et maudite! Tu nais pour la souffrance et l'humiliation. Enfanter dans les larmes et sans gloire ; te soumettre en silence, et te courber toujours, c'est là ton châtiment $\gg^{19}$. Le seul mot de réconfort pour l'asservie serait de «se résigner $\gg$ et d'avoir confiance en l'éternité après la vie terrestre. Ève découragée, pour ne pas dire, désespérée, cherche un appui auprès de la Société, coiffée du bonnet phrygien et enveloppée dans un drapeau tricolore. Celle que l'on considère comme généreuse puisqu'elle porte au front les mots d'ordre : « Liberté, Égalité, Fraternité $\gg$, ne semble point disposée à avoir pitié des larmes de la malheureuse. Ce personnage grotesque déclare que la liberté n'est pas pour tous et qu'elle n’a pas été inventée pour que la femme jouisse de ses attributs : « Fais ton devoir, ô femme ; accomplis ta besogne, sans te laisser distraire par d'inutiles rêves. Enfante, enfante, enfante ; il me faut des citoyens $\gg^{20}$. Abandonnée par tout le monde, la femme n'espère plus rien. C'est alors que l'on entend dans les coulisses un bruit et puis des phrases musicales de «L'Internationale $»$. Entre sur la scène la Révolte, drapée d'écarlate et les cheveux au vent. Fière et superbe, elle incite 《l'éternelle opprimée » à se lever des genoux et à marcher droit vers la justice. La « victime séculaire » subit une métamorphose sous l'effet du discours révolutionnaire de ce personnage et de femme soumise et résignée elle devient une agitatrice emportée. Dans son monologue accusateur, qui annonce les exhortations enflammées des expressionnistes, la première femme attaque ouvertement la religion et la société :

18 Cf. « L'esclavage de la femme, il est nécessaire, il est préparé, consacré par sa faiblesse et les infirmités de sa nature; elle reste, dans la réalité brutale, la femelle génératrice dont l'enfantement est la fonction. Elle subit les approches du mâle ; elle est, dans l'étreinte, celle qui supporte. Si donc elle veut vaincre, elle ne le pourra qu'en privant son vainqueur de tous les moyens de triomphe dont il abuse, en restant chaste. Qui faiblit, tombe, et qui tombe, doit se soumettre. Pas de faiblesse, pas de chute dans la matière ! Que la femme émancipée garde une horreur nerveuse et préservatrice pour l'acte sexuel, et conserve sa vierge cuirasse. Et si la vie, si l'ordre du monde comme l'ordre des sociétés, si la fatalité des lois cosmiques comme la fatalité des lois humaines veulent, rendent inévitables les misères de la femme ; si la femme ne peut pas être sans que l'amour charnel la domine et l'écrase, eh bien, périsse la vie elle-même », (M. Bigeaon, Les Révoltés Scandinaves, L. Grasilier, Éditeur, Paris, 1894, p. 178).

19 N. Roussel, Par la révolte, [in] Au temps de l'anarchie..., p. 356.

20 Ibidem, p. 357. 
Perfide Religion, Société infâme, barrière monstrueuse des préjugés et des sottises, votre esclave est une révoltée !... La prisonnière secoue les barreaux de sa prison ! (À l'Église.) Ah ! Tu parlais de châtiment ! (À la Société.) Et toi, tu parlais de sacrifice ! (Douloureusement.) Et pendant des siècles, toujours, les mêmes mots, lugubres, obsédants, ont frappé mes oreilles comme un glas ! (Terrible.) Taisez-vous, oppresseurs éternels ! Aujourd'hui, c'est de droits qu'il s'agit ! ${ }^{11}$

Dans son deuxième drame au titre parlant Pourquoi elles vont à l'église, Roussel renoue sans dissimulation avec les pièces à thèse : elle pose la question de la religiosité des femmes qui, pour une majorité écrasante, participent activement aux messes. Ce problème l'intéresse d'autant plus qu'elle-même a reçu une éducation catholique dont elle s'est détachée, surtout après son mariage avec Henri Godet. Mais à part la dévotion féminine, qui est sans aucun doute l'intrigue principale de son œuvre, l'auteure n'en attaque pas moins les hommes se prétendant libres-penseurs en ce qui concerne la lutte sociale et en même temps aveugles à la condition précaire des femmes. De fait, dans son unique pièce quotidienne, Roussel présente un couple dans lequel certains rôles, - on voudrait dire les « fonctions sociales »-sont bien répartis : le mari, simple employé, fréquente des réunions au cours desquelles on débat des problèmes des plus démunis. De prime abord, monsieur Bourdieu pourrait nous paraître bien sympathique, mais il gronde sa femme de ne pas savoir bien cuisiner et de mal servir son « maître ». Madame Bourdieu devrait, conformément aux sacro-saintes règles du masculinisme remplir les rôles d'une cuisinière, d'une bonne, d'une infirmière et de temps à autre celle d'une amante dévouée à son mâle. La dramaturge dénigre alors avec véhémence l'égoïsme de l'homme dont les idées progressistes restent en net contraste avec ses préjugés sur la nature féminine. Ce libertaire qui prône le triomphe de la morale laïque interdit à sa femme de se présenter à l'église. Et il ne le fait pas au nom de ses convictions idéologiques en la matière, mais parce que sa situation de vice-président de la Libre Pensée serait éclaboussée par un tel geste. Le prétendu révolutionnaire ne diffère point d'un bourgeois bien-pensant qui traite sa femme comme sa propriété. La femme abandonnée cherche de la consolation dans les bras du clergé qui, au demeurant, l'encourage à supporter avec « dignité » son destin d'éternelle inférieure. Voilà la thèse que Roussel défend dans son texte satirique (elle n'a pas tort de choisir cette tonalité qui lui permet d'être plus persuasive). Dans son drame, madame Bourdieu ira à l'église avec sa voisine (elle aussi athée) non pour chercher la « bonne nouvelle », mais pour des raisons plus triviales : elle s'ennuie seule dans la maison et le dimanche on peut se distraire, manger le pain bénit et s'empiffrer de brioches excellentes.

\footnotetext{
${ }^{21}$ Ibidem, p. 358.
} 
Quoi qu'il en soit, la pièce de Roussel, qui s'inscrit dans le combat anticlérical, illustre ses idées sur les méfaits de la religion, mais elle est loin d'inculper les femmes pour leur soumission prétendument innée :

Pour vaincre un mal, il faut en connaître la cause. Pour détourner les femmes de l'Église il faut comprendre pourquoi elles y vont. Et c'est toute la question féministe qui se pose ici devant nous. Elles y vont parce que tout - l'éducation qu'elles ont reçue, les mœurs, les institutions - leur en fait un devoir et surtout un besoin. C'est leur ignorance, leur humilité, leur désintéressement des réalités de la vie sociale, le vide de leur existence, leurs déceptions, leurs chagrins, leur désir de distraction, ou, au contraire, de paix et d'oubli, leur vague soif insatisfaite d'art, d'élévation, d'idéal, c'est tout cela qui les conduit et les agenouille au pied des autels.

Par un enchaînement curieux, mais logique, la religiosité des femmes, effet de l'injustice sociale dont elles souffrent, en est en même temps la cause ${ }^{22}$.

L'action du troisième drame La Faute d'Ève, qui recourt à l'allégorisation comme son premier texte, se déroule au paradis terrestre. Tout y est fait pour plaire aux habitants « élus » : pelouses vertes, cascades chantantes et partout des fleurs en massifs ou en guirlandes. Difficile de ne pas y penser autrement que comme à un décor charmant dont on affuble l'imagination pieuse du croyant rêvant d'une vie meilleure après celle qu'il mène péniblement sur terre. L'auteure semble ainsi dénigrer cet aspect puéril de la représentation sereine d'un monde fantasmagorique qui s'oppose d'une façon frappante à la réalité cruelle de l'existence morne au quotidien. Dès le début, nous assistons à une conversation entre Adam et Ève ; ce premier profite de la sérénité qui règne dans le paradis tandis que sa femme légitime s'y ennuie affreusement. Le mâle apprécie à sa juste valeur l'harmonieux enlacement des lignes et la beauté omniprésente dans ce jardin magnifique. Il ne comprend pas l'accablement de la femelle, pour qui le paradis monotone paraît plutôt un lieu de torture : «plus rien ne m'intéresse parce que je sens que plus rien ne se révélera davantage. Ces animaux trop dociles, aux yeux vides, ne m'amusent plus, je les méprise ; ce ciel toujours bleu pèse sur ma tête ; tant de sérénité m'accable, et la succession ininterrompue des jours pareils, des nuits semblables, est pour moi un supplice sans cesse renouvelé $\gg^{23}$. La seule chose qui l'intrigue dans le jardin merveilleux, c'est la fleur « défendue » de la science. Adam est terrifié ne serait-ce qu’à l'idée de penser à cueillir cette fleur. Il prévient sa compagne que le Seigneur a strictement interdit d'y songer. Cependant, la première femme prend quelque peu à la légère ces interdictions d'autant plus qu'elles lui semblent parfaitement déraisonnables.

22 N. Roussel, [in] La Libre-Pensée internationale, 15 juillet 1911.

23 N. Roussel, La Faute d'Ève, [in] Au temps de l'anarchie..., p. 380. 
Ainsi, sourde aux admonestations de son compagnon, elle arrache la fleur pour la humer. D'un coup elle s'exalte à l'arôme émanant de la plante : «comme il me grise !... Il me semble qu'autour de moi quelque chose s'entrouvre, et que je monte... Je monte... et tout se recule, tout s'élargit, tout se transfigure $! \gg^{24}$ L'enthousiasme ne dure pas longtemps, car suite à cette « faute » impardonnable on entend un roulement de tonnerre et un ange au visage sévère entre en scène, une épée flamboyante à la main, sûrement pour faire un peu plus peur. Adam recule ahuri, mais Ève affronte courageusement cet envoyé du bon Dieu. De fait, la femme n'est point effrayée par les mots de l'ange, selon lequel dès lors un châtiment va peser très lourdement sur toutes les femmes (il annonce que l'homme va souffrir, proportionnellement à son péché, ce qui veut dire, un peu moins que sa compagne), non seulement elle n'a pas peur de cette « instance céleste » privée de tout attribut angélique, mais elle se montre aguerrie à son égard. Sans prendre garde au discours menaçant de l'ange, elle se dresse contre la colère incompréhensible de Dieu. Elle-même est fâchée, car elle a compris qu'elle avait mené jusque-là une vie minable et artificielle, dorénavant elle est décidée à vivre réellement, indifférente aux prescriptions aussi absurdes que chimériques du Créateur, en dehors du paradis, ce qui veut dire qu'elle n'en est pas vraiment chassée, car c'est elle qui quitte cet Eden fallacieux sans le moindre regret. Elle tend la main à Adam et l'invite à l'accompagner dans son voyage vers la vraie vie. Ses mots, qui mettent fin à la pièce, font penser à elle comme à une nouvelle Ève, rédemptrice de toute l'humanité :

Viens vers les luttes qui meurtrissent, et les conquêtes qui enivrent ; viens vers l'angoisse qui torture, et vers l'amour qui console. Viens vers l'action et vers le rêve ; vers les ténèbres qui peu à peu s'éclairent, et vers les horizons qui toujours s'élargissent ; viens vers la découverte éternelle et le mystère sans cesse renaissant. Viens vers toutes les douleurs, vers tous les espoirs, vers tous les orgueils! Viens vers la vie enfin, énorme, tumultueuse! Viens! Viens! Nous n’avons pas encore vécu; nous allons vivre...25

\section{Conclusion}

Inspirée par Tolstoï, Zola et Ibsen, Vera Starkoff compose de vraies pièces à thèse dans lesquelles elle s'en prend au système désuet qui assujettit la femme à l'esclavagisme. Ses textes pèchent par leur didactisme parfois outrancier, mais nous devons toujours tenir compte du public peu féru de théâtre auquel elle s'adresse. La simplicité est son arme, redoutable, même si elle n'incite pas ouver-

24 Ibidem, p. 381.

25 Ibidem, p. 383. 
tement aux actes révolutionnaires. Elle expose ses thèses, propose des solutions et laisse le libre choix aux spectateurs qui devraient réfléchir sur les possibilités de remédier à la condition plus que précaire des femmes dans la société dominée par les hommes. Si Starkoff peut être considérée comme une auteure plutôt pondérée, alors sa compatriote Roussel s'avère plus offensive et blasphématoire. De fait, elle dénigre d'une manière véhémente les sacro-saintes valeurs représentées par l'Église et l'État qui oppressent non seulement les femmes mais tout individu aux idées personnelles. Elle ne fait pas dans la dentelle quand elle dénonce la tradition judéo-chrétienne qui légifère toutes les injustices sociales en réduisant le rôle subalterne de la femme à l'accouchement des enfants. Quoique née dans une famille bourgeoise, la dramaturge rejette aussitôt les dogmes antisociaux de cette classe dominante et surtout se lance dans son théâtre comme une anticléricale incorrigible. C'est pourquoi elle a recours à la tradition du théâtre médiéval qu'elle modernise d'une manière originale. Ses pièces allégoriques, exemples des moralités à rebours, invitent à se soulever contre les fantasmes religieux, imaginés par la gent masculine, selon lesquels la femme était considérée comme pécheresse et en conséquence devait être traitée comme inférieure par rapport à l'homme. Roussel se rebelle tout en espérant qu'un jour tous les êtres humains seront égaux. Peu importe que sa voix puisse sembler utopique, par sa rigueur et sa sévérité quelque peu blessante, elle encourage d'autres camarades à combattre pour un avenir plus juste envers le sexe faible. Le théâtre féministe est en ébullition. Il en est de même dans les cercles des militantes. Les femmes sont enfin passées à l'attaque. Que cette offensive soit intransigeante, les propos de Madame Valbrègue, avocate à la Cour d'appel et qui ne mâche pas ses mots, en témoignent. Elle déclare avec une malice ostentatoire :

Les détracteurs du féminisme, et en particulier beaucoup de médicastres - j’allais dire médecins - déclarent que la condition physiologique de la femme - je veux dire son état périodique - la met dans un état d'infériorité intellectuelle... En opposition à leurs assertions, laissez-moi vous demander si l'homme n'est pas lui-même, de par sa condition physiologique, dans un état d'infériorité par rapport à la femme. Eh bien, je déclare que oui.

Prenons l'homme, ou le jeune homme à peine pubère. N'a-t-il point l'obsession de l'amour au point de vue physiologique, et les parents ne disent-ils pas : il faut qu'il jette sa gourme ? Ce n'est pas au point de vue social ni au point de vue moral que je parle ; je parle au point de vue scientifique et au point de vue de l'opposition des faits ; le jeune homme n'a-t-il pas, dès qu'il est pubère, l'obsession de l'amour ? L'homme adulte ne continue-t-il pas ? Et le vieillard lui-même ne cherche-t-il pas, par toutes sortes de moyens aphrodisiaques, à revivre ses années d'adulte, son passé ? Eh bien, oui ou non, est-ce que lorsque l'homme est possédé de ce désir et de cette obsession, il n'est pas dans un état d'infériorité patent ? A-t-il d'autre objectif que la possession, par tous les moyens possibles, de la femme désirée ? Et comme 
cela lui arrive plus d'une fois par mois, peut-être une fois par jour, n'est-il pas, lui, d'une façon pour ainsi dire permanente, dans un état physiologique inférieur à celui où se trouve la femme accidentellement ? ?6

\section{The voice of women in the French theater of contest at the turn of the twentieth century}

Until recently not many people recognize the feminist theater movement existing in the end of the $19^{\text {th }}$ century. For almost a century it was condemned to oblivion for above all political reasons. Most scholars were focused on anarchist male authors while in this the article works of the two feminine authors are analyzed, these include: Vera Starkoff and Nelly Roussel. Starkoff, the Russian Jew, was inspired by Tolstoy, Zola and Ibsen, and wrote the didactic dramas in which she discussed gender inequality between men and women at that time. She in her works seems to be more moderate then Roussel who is more uncompromising in her works. Roussel ridicules canonic values represented by the Roman-Catholic Church enslaving not only women but each individual's thinking.

Keywords: Vera Starkoff - Nelly Roussel - feminist theater - anarchy - didactic drama Mots-clés: Vera Starkoff - Nelly Roussel - théâtre féministe - anarchie - drame didactique

${ }^{26}$ Propos de M. Valabrègue [in] (réd. Oddo Deflou), Congrès national des droits civils et du suffrage des femmes, tenu en l'Hôtel des Sociétés Savantes à Paris les 26, 27 et 28 juin 1908, Paris, 1911, p. 180. 